\title{
Psicooncología
}

ISSN: 1696-7240

\section{Padres diagnosticados de cáncer: malestar emocional y recursos para comunicarse con sus hijos menores}

\author{
Sònia Fuentes ${ }^{1, *}$; Tomás Blasco ${ }^{2}$
}

Recibido: 30 de mayo de 2017 / Aceptado: 25 de agosto de 2017

Resumen. Objetivo: Conocer los recursos que poseen los padres afectados por cáncer al comunicar la enfermedad a sus hijos menores y su relación con el malestar emocional de estos pacientes. Método: Se evaluó el estilo de comunicación familiar mediante una subescala del FAD (Mc Master Family Assessment Device). Se elaboró un cuestionario específico para evaluar los recursos de los pacientes, donde cada ítem era valorado con una escala de 0 a 10 (siendo 0 nada en absoluto y 10 , la máxima puntuación posible). También se utilizó una escala numérica de valoración del malestar emocional, en forma de un solo ítem (0-10). El conjunto de pruebas se aplicó a 60 pacientes de edades comprendidas entre los 34 y los 60 años todos ellos padres afectados de cáncer con hijos menores a su cargo. Resultados: Los recursos que parecen poseer en mayor medida los padres evaluados son los recursos relacionados con la gestión de la vida doméstica (media $=8,3$ ), seguidos de los que hacen referencia a delegar a terceras personas los cuidados de sus hijos durante momentos difíciles. Al analizar con más profundidad estos dos recursos, detectamos que más del $80 \%$ de los padres de la muestra poseen el recurso relacionado con la gestión de la vida doméstica. Por el contrario, el recurso de como informar sobre la situación de enfermedad a los niños, lo tiene poco más de la mitad de los pacientes evaluados (55\%). Los datos indican que existe una relación inversa entre la disponibilidad de este recurso y el nivel de malestar emocional, ya que los pacientes que consideran tener recursos para hablar de la enfermedad a sus hijos, presentan un menor malestar emocional. Conclusiones: Los padres recién diagnosticados de cáncer que plantean comunicar la situación de enfermedad a sus hijos menores, poseen más recursos para gestionar la vida doméstica y delegar el cuidado de sus hijos cuando lo precisan a terceras personas, que para informar sobre la enfermedad y sostener emocionalmente a sus hijos. Así mismo, el no disponer de recursos para comunicar el diagnóstico a los hijos se asocia con una mayor presencia de malestar emocional.

Palabras clave: Comunicación en cáncer; recursos psicológicos; padres enfermos; hijos; malestar emocional.

\section{[en] Parents receiving cancer diagnosis: emotional distress and resources to communicate with their children}

Abstract. Objective: To know which resources to communicate the illness to their children and whether these resources are related with emotional distress in cancer patients at diagnosis. Method: Sixty patients aged 34-60 years, who have been recently diagnosed and had children less of 18 years old reported their

1 Unidad de Psico-Oncología. Institut Català d’Oncologia. Hospital Universitari Germans Trias i Pujol. Badalona. Hospital de Mataró.

E-mail: sfuentes@iconcologia.net

2 Grupo de Investigación en Estrés i Salud GIES. Unitat de Psicologia Bàsica. Universitat Autònoma de Barcelona. E-mail: tomas.blasco@uab.es

Dirección de Correspondencia: Sònia Fuentes Sanmartín.Unidad de Psicooncologia. Instituto Catalán de Oncología - Badalona. Hospital Universitario Germans Trias i Pujol. Carretera del Canyet, s/n.08916 - Badalona. E-mail: sfuentes@iconcologia.net 
resources, emotional distress and family communication style. Family communication was assessed using the specific subscale of the FAD (McMaster Family Assessment Device). Patients resources were assessed with a specific questionnaire whose items were valued in a $0-10$ scale (being 0 nothing and 10 the highest punctuation possible). Emotional distress was assessed with a numeric scale ranging 0-10. Results: Resources more available were those related with the management of daily life (mean= 8.3), followed by resources to delegate to others the care of their children. Both resources were available for more than a $80 \%$ of patients. However, resources related with telling the children the illness situation, were available to only $55 \%$ of patients. Patients with resources to communicate the illness situation to their children reported less emotional distress. Conclusions: Parents who were recently diagnosed with cancer have more resources to manage their daily life and to delegate the care of their children to others, than to inform them about the illness and to help them to cope emotionally. Parents with less resources to communicate with their children show a higher level of emotional distress.

Keywords: Cancer communication; psychological resources; ill parents; sons; emotional distress.

Sumario. 1. Introducción 2. Método 3. Análisis estadísticos 4. Resultados 5. Discusión 6. Agradecimientos 7. Referencias bibliográficas.

Cómo citar: Fuentes S, Blasco T. Padres diagnosticados de cáncer: malestar emocional y recursos para comunicarse con sus hijos menores. Psicooncología 2017;14:229-240. Doi: 10.5209/PSIC.57082.

\section{Introducción}

Desde hace años, en el campo de la psiquiatría y la psicología infantil ${ }^{(1)}$, se ha ido investigando sobre cómo ayudar a los niños y adolescentes durante el proceso de duelo. Desde los cuidados paliativos, también se han realizado estudios y documentos específicos de soporte a las familias, focalizados básicamente en el fin de la vida de alguno de los padres u otro familiar cercano. En este aspecto, es amplia la bibliografía de soporte a los padres que se encuentran afrontando la pérdida de un ser querido ${ }^{(2,3)} \mathrm{y}$ abundantes los estudios realizados al respecto desde esta especialidad ${ }^{(4)}$. Durante los últimos años, la sociedad ha asumido los aspectos relacionados con la proximidad de los niños y adolescentes a los procesos de pérdida, especialmente ante la muerte de un ser querido. Es abundante la bibliografía en forma de cuentos y libros de soporte, dirigido a este colectivo y a los padres que les hacen de guía.

De todas formas en las sociedades occidentales, las sociedades del norte, sigue siendo frecuente mantener a los niños, al margen de los procesos naturales que forman parte de la vida, como ocurre con el envejecimiento, el deterioramiento, la enfermedad y la muerte. En este contexto, no es extraño entender las dificultades de comunicación que pueden presentarse ante un proceso de pérdida y que varían según la edad del menor, el tipo de personalidad que este desarrolle, la maduración psicoafectiva en el momento de la pérdida, el vínculo con la persona fallecida, etc., entre otros muchos aspectos a tener en cuenta ${ }^{(5)}$.

Estos procesos también se ven amenazados durante el desarrollo de la enfermedad oncológica, por la presencia constante de la incertidumbre relacionada con una posible muerte cercana. Estas situaciones de enfermedad implican cambios, tales como alteraciones en la imagen corporal de la persona afectada y también importantes dificultades en el proceso de comunicación del adulto con cáncer, tanto hacia otros adultos como hacia los niños, especialmente en el caso de los hijos ${ }^{(6)}$. A todo esto hay que añadir que a los adultos, hablar sobre estas situaciones les comporta una 
cierta incomodidad, les es dificultoso y, a menudo lo evitan hacer entre ellos. No nos debe extrañar pues, que eviten hablar de estos temas con los niños que viven a su alrededor y con los cuales mantienen algún tipo de vínculo afectivo.

Si bien los recursos de soporte a los padres que tienen que enfrentar situaciones de fin de vida son habituales en nuestros entornos, los estudios focalizados en otros momentos de la enfermedad y concretamente, en las preocupaciones de los padres recién diagnosticados de cáncer y en las dificultades o alteraciones en el proceso de comunicación entre los padres enfermos y sus hijos, han sido escasos, hasta que en los últimos cinco años han aparecido investigaciones relacionadas con el tema ${ }^{(6-8)}$.

Lamentablemente en la práctica clínica oncológica, al igual que ocurre en nuestra sociedad, un importante número de pacientes padres recién diagnosticados de cáncer, se ven a menudo sobrepasados en el momento de decidir cómo comunicar a sus hijos la situación de enfermedad ${ }^{(9,10)}$. Esta situación puede llegar a generar un importante malestar emocional en la persona afectada y en su entorno familiar, o bien incrementar el malestar ya existente provocado por la situación oncológica, teniendo en cuenta que para la persona que lo sufre, es un momento de difícil gestión psicológica, ante el nuevo diagnóstico y la toma de decisiones que este comporta ${ }^{(11)}$. Frecuentemente, esta es una preocupación expresada, por parte de los pacientes afectados, a los oncólogos o enfermeras consultoras que los atienden en el momento del inicio del proceso. Al respecto cabe decir que, hasta hace poco, este era un tema poco abordado en la literatura científica ${ }^{(7,12)}$ que a lo largo del tiempo, se ha decantado más por el estudio de la afectación psicológica de los hijos ante el cáncer de los padres ${ }^{(13-18)}$. No obstante, en una revisión más reciente ${ }^{(19)}$, ya ponen de manifiesto esta necesidad detectada en los pacientes y en las familias sobre como comunicar malas noticias a los más pequeños, si bien, este trabajo se centra en el momento inmediato tras el fallecimiento del paciente progenitor, tal como se ha señalado anteriormente.

Hay muy pocos estudios que analicen y/o expliquen de qué depende que los padres diagnosticados de cáncer, comuniquen con mayor o menor dificultad o estrés la enfermedad a sus hijos ${ }^{(7,17)}$. Posiblemente, la falta de recursos para manejar la situación en su conjunto y un estilo de comunicación familiar preexistente puedan ser aspectos que determinen este malestar emocional. De ahí que el presente estudio aborde el análisis de los recursos de estos pacientes en el momento concreto del diagnóstico de la enfermedad. La razón para elegir ese momento es porque consideramos que es un momento crucial para iniciar los diálogos con los hijos. De esta manera si, en un futuro hay una recaída de la enfermedad, tanto a la persona afectada como a otros miembros de la familia les serán de ayuda, las pautas a seguir que ya utilizaron en el momento del diagnóstico de la enfermedad y que por desgracia, de nuevo, tienen que volver a utilizar. En este sentido cabe destacar la aportación pedagógica y de soporte de algunos autores, que han facilitado un soporte bibliográfico específico para los padres que se encuentran en estas situaciones y para sus niños. Parte de esta bibliografía de soporte se encuentra en formato específico paro los más pequeños en forma de cuento ${ }^{(20-22)}$, siendo estos de gran ayuda a los pacientes, ante un nuevo diagnóstico y/o una recaída de su enfermedad oncológica.

Dentro de este contexto, el objetivo del trabajo es conocer los recursos que poseen los padres recién diagnosticados de cáncer para comunicar la situación a sus hijos menores de edad, y la relación que pueden tener estos recursos con el malestar emocional que experimentan esos padres. 


\section{Método}

\subsection{Participantes}

La muestra está formada por 60 participantes que habían recibido el diagnóstico en las últimas semanas, realizaban la primera visita en Consultas Externas de Psicooncología, eran padre o madre con hijos menores de edad a su cargo y aceptaron participar voluntariamente en el estudio. Se reclutaron en seis centros hospitalarios de Cataluña (ver Tabla 1). Un $85 \%$ de la muestra eran mujeres, con una media de edad de 43,8 años $(\mathrm{SD}=5,30)$. El resto de características de los participantes se indica en la tabla 1

Tabla 1. Características de los participantes en el estudio.

\begin{tabular}{|c|c|c|c|}
\hline \multirow[t]{2}{*}{ Género } & Mujer & 51 & 85,00 \\
\hline & Hombre & 9 & 15,00 \\
\hline \multirow[t]{6}{*}{ Hospital } & ICO Bad & 30 & 50,00 \\
\hline & HSJC & 11 & 18,33 \\
\hline & BSA & 7 & 11,67 \\
\hline & HES & 4 & 6,67 \\
\hline & HGG & 4 & 6,67 \\
\hline & ICO Gi & 4 & 6,67 \\
\hline \multirow[t]{4}{*}{ Estadio Enferm. } & I & 7 & 11,67 \\
\hline & II & 18 & 30,00 \\
\hline & III & 25 & 41,67 \\
\hline & IV & 10 & 16,67 \\
\hline \multirow[t]{3}{*}{ Pronostico Enferm. } & Bueno & 23 & 38,33 \\
\hline & Malo & 14 & 23,33 \\
\hline & Reservado & 23 & 38,33 \\
\hline \multirow[t]{2}{*}{ Rol Familiares } & Padre & 9 & 15,00 \\
\hline & Madre & 51 & 85,00 \\
\hline \multirow{4}{*}{ Tipo Familia } & Tradicional & 46 & 76,67 \\
\hline & Monoparental & 11 & 18,33 \\
\hline & Reconstituida & 2 & 3,33 \\
\hline & Otros & 1 & 1,67 \\
\hline \multirow[t]{3}{*}{ Origen cultural } & Español & 55 & 91,67 \\
\hline & Europeo & 3 & 5,00 \\
\hline & País latino & 2 & 3,33 \\
\hline \multirow[t]{4}{*}{$\mathrm{N}^{\mathrm{o}}$ hijos } & 1 & 16 & 26,67 \\
\hline & 2 & 39 & 65,00 \\
\hline & 3 & 4 & 6,67 \\
\hline & 4 & 1 & 1,67 \\
\hline
\end{tabular}

\section{Hospitales:}

ICO Bad: Instituto Catalán de Oncologia Badalona HSJC: Hospital St. Jaume de Calella BSA: Hospital Municipal de Badalona HES: Hospital Espiritu Santo Sta. Coloma de Gramanet HGG: Hospital General de Granollers ICO Gi: Instituto Catalán de Oncologia Girona 


\subsection{Material}

- Hoja de Recogida de Datos Médicos: con el objetivo de recoger datos clínicos se elaboró un formulario de datos de cada paciente donde se hacían constar los siguientes: edad del/la paciente; diagnóstico, estadio de la enfermedad y pronostico (respecto al diagnóstico oncológico), rol familiar, tipo de familia y origen cultural de la misma, y número de hijos y edad de los mismos.

- Estilo de comunicación familiar: Se utilizó la subescala de comunicación del FAD (Mc Master Family Assessment Device) ${ }^{(23)}$. Para ello, los autores del presente trabajo realizaron una traducción de los 9 ítems que la componen, aplicándolos a otra muestra compuesta por 29 pacientes $^{(24)}$ y obteniendo una fiabilidad satisfactoria (con un valor de 0,75 en el alfa de Cronbach, similar al valor 0,72 reportado por los autores de la escala original). Los ítems se responden mediante una escala Lickert de 4 puntos $(1=$ Completamente de acuerdo; 2=De acuerdo; $3=$ En desacuerdo; 4=Completamente en desacuerdo), calculándose el promedio como valor final. Según los autores del FAD, puntuaciones superiores a 2 indican que existe una comunicación disfuncional en la familia. El valor alfa de Cronbach obtenido en la muestra del presente estudio fue de 0,81 .

- Recursos para comunicar a los niños/adolescentes la situación de enfermedad: Este aspecto se valoró mediante la creación de cuatro ítems específicos mediante los cuales los padres valoraban en qué grado poseían determinadas estrategias para manejar la situación creada por la enfermedad: a) Poder ser capaz de informar de la enfermedad a los hijos; b) Poder sostener emocionalmente el impacto que la comunicación de la enfermedad genera en los hijos; c) Poder gestionar la vida escolar y doméstica, y: d) Poder obtener de otras personas la ayuda necesaria para manejar la situación. Todos los ítems se evaluaban mediante una escala numérica 0-10 (donde " 0 " indica "ningún recurso", y "10" indica "muchos recursos"). Se consideró que dos de estos recursos dependían exclusivamente de las propias capacidades del paciente: "Capacidad para Informar" y "Capacidad para sostener emocionalmente", mientras que los otros dos dependían parcial o totalmente de las características del entorno familiar: "Gestionar la vida doméstica" y "Poder obtener ayuda de otras personas". A fin de establecer dos niveles respecto a la disponibilidad de recursos, se consideró que estos eran satisfactorios cuando la puntuación en la escala era igual o mayor a 7, e insuficientes cuando la puntuación en la escala era inferior a ese valor.

- Escala de valoración del malestar emocional: Se utilizó la pregunta (¿Qué malestar emocional sientes en el momento presente?) evaluada mediante una escala numérica 0-10, de forma similar al Distress Thermometer. Teniendo en cuenta estudios recientes ${ }^{(25-27)}$, se estableció el punto de corte en el valor 6 para considerar que la persona presentaba un malestar emocional elevado. De este modo, los pacientes se clasificaron en dos grupos: pacientes con malestar emocional elevado (valores superiores a 6 en el ítem) o sin malestar emocional elevado (valores iguales o inferiores a 6 en el ítem).

\subsection{Procedimiento}

El estudio fue autorizado por el CEIC (Comité de Ética e Investigación Clínica) del Hospital Universitario Germans Trias i Pujol. La recogida de datos se llevó a cabo 
desde febrero de 2012 hasta febrero de 2014 en los seis hospitales indicados en la Tabla 1. Los pacientes eran atendidos en la primera visita de Consultas Externas de Psicooncología. Al final de la misma, en aquellos casos que cumplían los criterios de inclusión, se les solicitaba la colaboración para participar en el estudio. Si aceptaban, se les administraba el consentimiento informado y a continuación, los cuestionarios y preguntas descritos en el apartado de Material y en el siguiente orden: Escala de Comunicación Familiar, Recursos para comunicar la enfermedad, y Grado de Malestar Emocional. Después, y a partir de la historia clínica del paciente, la psicooncóloga anotaba en la Hoja de Recogida de Datos Médicos la información requerida.

\section{Análisis estadístico}

Todos los análisis se realizaron con el programa estadístico SPSS (versión 18.0). Los datos descriptivos se muestran con medias, desviaciones estándar, rangos y distribución de frecuencias.

Para la comparación entre variables dicotómicas utilizamos la prueba de Chi cuadrado. La significación se ha considerado con un nivel de probabilidad del $5 \%$ o inferior y se indica la significación exacta proporcionada por el programa estadístico.

\section{Resultados}

Respecto a la disponibilidad de recursos, la Tabla 2 muestra que el que está disponible en mayor medida es el relacionado con la gestión de la vida doméstica, mientras que los recursos menos disponibles son los relacionados con informar a los hijos y el sostenerlos emocionalmente. Utilizando el criterio de existencia satisfactoria de los recursos, puede observarse (Tabla 3) que más del $80 \%$ de los pacientes disponían de recursos para gestionar la vida doméstica, pero menos del $60 \%$ podían ser capaces de informar de la enfermedad a los hijos o de sostenerlos emocionalmente.

E1 40\% de los pacientes muestra tener un estilo comunicativo disfuncional, ya que mostraban una puntuación superior a 2 en la subescala del FAD. El tipo de estilo comunicativo no guardaba relación con el nivel de recursos de los pacientes.

Tabla 2. Recursos de los padres participantes en el estudio.

\begin{tabular}{lcc}
\hline & Media & DS \\
\hline Gestionar vida doméstica & 8,13 & 2,49 \\
Delegar a terceras personas & 7,40 & 3,20 \\
Sostener emocionalmente & 6,77 & 2,64 \\
Informar de la enfermedad & 6,45 & 2,90 \\
\hline
\end{tabular}


Finalmente, 40 pacientes $(66,7 \%$ de la muestra) tienen un nivel de malestar emocional elevado (puntuación superior a 6 en la Escala de Malestar Emocional.

Tabla 3. Frecuencia de pacientes que expresan tener recursos suficientes (por encima del punto de corte 7).

\begin{tabular}{llcc}
\hline & Recursos & $\mathbf{N}^{\mathbf{0}}$ Pacientes & $\mathbf{\%}$ \\
\hline Recursos internos & Informar de la enfermedad & 33 & 55,00 \\
& Sostener emocionalmente & 35 & 58,33 \\
\hline \multirow{2}{*}{ Recursos externos } & Gestionar vida doméstica & 49 & 81,67 \\
& Delegar a terceras personas & 41 & 68,36 \\
\hline
\end{tabular}

En el análisis del malestar emocional, considerado éste en los dos niveles indicados en el apartado de Material, se observó que no guardaba relación con el estadio y pronóstico de la enfermedad. Tampoco con la edad de los pacientes ni con la de los hijos, así como tampoco con el estilo de comunicación familiar. Respecto a los recursos, el malestar emocional no se relaciona con la disponibilidad de recursos para gestionar la vida doméstica, pedir ayuda a otras personas, o sostener emocionalmente a los hijos. Sin embargo, sí está relacionado con disponer del recurso de informar a los hijos sobre la enfermedad (Tabla 4), ya que el porcentaje de padres que dicen poseer este recurso y no presentan malestar emocional elevado es superior al de los padres que no poseen este recurso $\left(\mathrm{X}^{2}=4,848 ; \mathrm{p}=0,028\right.$, Existencia del recurso para informar de la enfermedad y presencia de malestar emocional.

Tabla 4. Existencia del recurso para informar de la enfermedad y presencia de malestar emocional.

\begin{tabular}{lcccc}
\hline & \multicolumn{2}{c}{ Sin malestar emocional } & \multicolumn{2}{c}{ Con malestar emocional } \\
\cline { 2 - 5 } & $\mathbf{N}$ & $\mathbf{\%}$ & $\mathbf{N}$ & $\mathbf{\%}$ \\
\hline $\begin{array}{l}\text { Sin los recursos de informar a los hijos } \\
\text { de la enfermedad }\end{array}$ & 5 & 18,50 & 22 & 81,50 \\
\hline $\begin{array}{l}\text { Con los recursos de informar a los hijos } \\
\text { de la enfermedad }\end{array}$ & 15 & 45,50 & 18 & 54,50 \\
\hline
\end{tabular}

\section{Discusión}

Las ansiedades y preocupaciones de las personas que atraviesan una enfermedad oncológica son múltiples. Cuando estas personas tienen hijos menores a su cargo y presentan dificultades en el proceso de comunicación con sus hijos, es adecuado 
realizar una valoración psicooncológica. El presente estudio ha intentado aportar información sobre el perfil de recursos de que disponen los padres para abordar esta situación, a fin de guiar la forma de detectar y gestionar esta problemática. Nuestros datos indican que los pacientes en general, parecen disponer de suficientes recursos externos, como el delegar a terceras personas el cuidado de los hijos y la gestión de la vida doméstica; pero más de un $40 \%$ de los padres reconoce no disponer de los recursos suficientes para informar de la enfermedad a los hijos y/o para darles un apoyo emocional frente a la situación. Si bien no hemos localizado otros trabajos que nos permitan determinar si este porcentaje se da también en otras poblaciones dentro y fuera de nuestro entorno cultural, podemos afirmar que existe un importante volumen de pacientes que tiene esta problemática y por tanto, que puede beneficiarse de estrategias de intervención psicooncológica y programas de apoyo, cuya eficacia ha sido evidenciada en algunos trabajos ${ }^{(28-31)}$. También nos parece relevante destacar que la existencia de recursos no guarda relación con las variables médicas y sociodemográficas consideradas en esta investigación. No obstante, no puede afirmarse que esta relación no exista y sería necesario realizar más estudios que confirmasen o matizasen lo aportado por los datos de nuestro trabajo.

Por otra parte, nos parece relevante que no exista una relación entre el poseer o no recursos para informar de la enfermedad a los hijos y el estilo de comunicación familiar, pues cabría pensar que los padres que no poseen estos recursos, formarían parte de las familias que tuvieran un estilo de comunicación disfuncional, tal como sugiere la literatura ${ }^{(32,33)}$. El hecho de que nuestros resultados no confirmen esta relación podría deberse a que la escala de evaluación de la comunicación que hemos utilizado no fuera válida para situaciones de enfermedad grave o que la estructura de los ítems generara algún tipo de sesgo en la valoración, pues detectamos que algunos de ellos eran difíciles de entender y su sentido había de ser clarificado por la psicooncóloga. Tampoco cabe descartar que la relación entre el estilo comunicativo y la disponibilidad de recursos pudiera estar moderada por la gravedad de la enfermedad en el momento del diagnóstico. Así, podría ocurrir que, en una familia con un estilo de comunicación adecuado, éste fuera un recurso que redujera las dificultades para informar a los hijos de un diagnóstico que tiene buen pronóstico. Pero también podría ocurrir que este recurso fuera insuficiente $\mathrm{y}$, por lo tanto, que no redujera las dificultades de comunicación cuando se tuviera que informar de un mal pronóstico. Sería necesario realizar más estudios en otras muestras de pacientes oncológicos para determinar si, efectivamente, lo que evalúa la subescala del FAD sobre comunicación familiar es válido para este tipo de pacientes y si además, dicha validez puede venir determinada por la gravedad del pronóstico.

Cabe preguntarse si es la falta del recurso para informar a los niños lo que determina el malestar emocional del adulto. Sin embargo, nuestro estudio no permite inferir relaciones causales y sólo muestra la interrelación entre la disponibilidad del recurso y la adaptación del paciente a la enfermedad (entendida como el nivel de malestar emocional experimentado). Una adaptación más difícil, que podría estar producida por la existencia de un peor pronóstico, coincide con una menor disponibilidad del recurso para informar, por lo que cabe pensar que tal vez un mayor malestar emocional pueda impedir al paciente movilizar un recurso para informar que, en cambio sí posee ${ }^{(34)}$. En este último caso, una intervención de 
apoyo a los padres, en la línea de las sugeridas anteriormente, puede facilitar que el recurso se active y, de esta manera, se pueda reducir la magnitud de este problema y ello ayude a reducir también el malestar emocional del paciente. No debe olvidarse que nuestros datos han sido obtenidos en el momento del diagnóstico, cuando los niveles de malestar emocional suelen alcanzar los valores más elevados ${ }^{(35)}$.

Dentro de las limitaciones de este trabajo que especificamos más adelante, estos resultados permiten sugerir que parece adecuado que el abordaje del recurso relacionado con la capacidad para comunicarse e informar a los hijos sea un punto de partida para el apoyo psicooncológico, pues permite que los niños entiendan mejor la naturaleza de sus propias emociones y las de los adultos con los que conviven y con los que se sienten fuertemente vinculados ${ }^{(36)}$.

Consideramos que los resultados de nuestro trabajo aportan elementos importantes que pueden tener relevancia para el desarrollo de intervenciones psicooncológicas adecuadas, pero que deben ser matizados a la luz de las diversas limitaciones de la presente investigación. En primer lugar, la muestra obtenida es limitada por lo que a género se refiere. Somos conscientes de la gran necesidad de realizar estudios relacionados con el sentir del género masculino. En segundo lugar, la muestra también es limitada en cuanto al tipo de enfermedad oncológica, pues la mayor parte de ella hace referencia a pacientes con cáncer de mama. Deseamos transmitir la necesidad de abrir este tipo de estudios a otros diagnósticos oncológicos y muy especialmente a las enfermedades onco-hematológicas. Por otro lado, ya hemos señalado anteriormente las dificultades que puede comportar la evaluación del estilo de comunicación familiar y quizá sea necesario desarrollar otras estrategias para valorar con más precisión este aspecto de la relación entre padres e hijos, sin olvidar que quizá deban realizarse estudios en los cuales las muestras sean más homogéneas en cuanto a la gravedad del pronóstico, dado que ésta podría afectar al estilo de comunicación familiar, pese a que nuestros datos no confirman esa relación. Futuros estudios en nuestro medio cultural deberían contemplar todos esos aspectos para tratar de superar las limitaciones de nuestro trabajo.

Finalmente, creemos que pese a las limitaciones de la investigación y la natural cautela con que deben interpretarse los resultados, nuestros datos apoyan la necesidad de explorar en la consulta psicooncológica la relación entre el paciente y sus hijos menores de edad y ofrecer apoyo psicológico especialmente dirigido a facilitar la comunicación con los mismos en el caso de pacientes recién diagnosticados, puesto que un porcentaje importante de estos, carece de los recursos necesarios para hacerlo en ese preciso momento. Esto puede ayudar, no sólo a facilitar el proceso comunicativo y conseguir un entorno familiar mejor preparado para afrontar las dificultades e incertidumbres que conllevará la fase de tratamiento, sino también, probablemente, a reducir el malestar emocional del paciente y de este modo, facilitar su adaptación a la enfermedad, optimizando la eficacia de la visita psicooncológica ${ }^{(37)}$.

\section{Agradecimientos}

Deseamos expresar nuestro especial agradecimiento a las Dras. Milagros Bárez y Marisa de Cáceres, así como a Lydia Caba, Ana del Moral y Carmen Cortés, por su colaboración y su apoyo. 


\section{Referencias bibliográficas}

1. Gamo Medina E, Pazos Pezzi P. El duelo y las etapas de la vida. Rev Asoc Esp Neuropsiq 2009;29: 455-69.

2. Kroen WC. Cómo ayudar a los niños a afrontar la pérdida de un ser querido - Ed. Oniro S.A. Barcelona. 2002.

3. Lacasta M.A. Soler M.C. El duelo: prevención y tratamiento del duelo patológico. Cuidados después de la muerte Manual SEOM de Cuidados Continuos. 713-30. Dispublic S.L. 2004; Madrid.

4. Fearnley R. Death of a parent and the children's experience: Don't ignore the elephant in the room. J Interprof Care. 2010; 24:450-9. doi: 10.3109/13561820903274871.

5. Rodríguez, A. M. Els nens i adolescents davant el càncer avançat o terminal dels pares. (Tesi Doctoral). Barcelona: Universitat Autònoma de Barcelona. 2013.

6. Inhestern y Bultmann, J.C. Understanding parenting concerns in cancer survivors with minor and young-adult children. J Psychosom Res 2016; 87: 1-6. doi: 10.1016/j. jpsychores.2016.05.008.

7. Semple C, McCaughan E. Family life when a parent is diagnosed with cancer: impact of a psychosocial intervention for young children. Eur J Cancer Care 2012; 22: 219-31. doi. 10.1111/ecc. 12018

8. Lewis F, Casey S, Brandt P, Shands ME, Zahlis EH. The enhancing connections program: pilot study of a cognitive-behavioral intervention for mothers and children affected by breast càncer. Psychooncology 2005; 15: 486-97.

9. Fuentes S, Blasco T. Preocupaciones de los pacientes con cáncer de mama que reciben atención psicooncológica. Psicooncología 2010;7: 51-60.

10. Fuentes S, Blasco T. Factores predictores de la adaptación a la enfermedad en pacientes recién diagnosticadas de cáncer de mama que acuden a un servicio de psicooncología. Anal Psicol 2012; 28:736-42. doi:10.6018/analesps.28.3.156031

11. Arber,A. 'Think Family Project' International Conference on Cancer Nursing Vancouver, BC Canada; 2015.

12. Sordo T, Rubio I, Peralta M, Ángeles M, Ospina L. ¿Cómo se lo digo a mi hijo/a? Psicooncologia 2014; 11(supl. 1):1-83.

13. Thastum M, Watson M, Kiembarcher C, Piha J, Steck B, Zachariae, et al. Prevalence and predictors of emotional and behavioral functioning of children where a parent has a cancer: a multinational study. Cancer. 2009; 115: 4030-9. doi: 10.1002/cncr.24449.

14. Huizinga G, Visser A, Zelders-Steyn Y, Teule J, Reijneveld S, Roodbol P. Psychological impact of having a parent with cancer. Eur J Cancer 2011;47: 239-46. doi: 10.1016/ S0959-8049(11)70170-8.

15. Krattenmacher T, Kuhne F, Ernst J, Bergelt C, Romer G, Moller, B. Parental cancer: factors associated with children's psychosocial adjustment a systematic review. J Psychosom Res 2012; 72:344-56. doi: 10.1016/j.jpsychores.2012.01.011

16. Krattenmacher T, Kühne F, Führer D, Beierlein V, Brähler E, Resch F, et al. Coping skills and mental health status in adolescents when a parent has cancer: A multicenter and multi-perspective study. J Psychosom Res 2013; 74, 252-9. doi: 10.1016/j. jpsychores.2012.10.003

17. Phillip, F. Adolescents living with a parent with advanced cancer: a review of the literature. Psychooncology 2014;23:1323-39. doi: 10.1002/pon.3570 
18. Ellis SJ, Wakefield CE, Burns M. Supporting children facing a parent's cancer diagnosis: a systematic review of children's psychosocial needs ans existint interventions. Eur J Cancer Care 2017; 26 (1). doi: 10.1111/ecc.12432.

19. Mesquida V, Seijas R, Rodríguez M. Los niños ante la pérdida de uno de los progenitores: revisión de pautas de comunicación eficaces. Psicooncologia 2015;12:417-29. doi. 10.5209/rev_PSIC.2015.v12.n2-3.51019

20. Britz R. El álbum de mamá. Barcelona . Novartis Oncology, 2014.

21. Elizegi, Rebeka. Una flor de recanvi per a la mare. Barcelona: Editorial Takatuka, 2011.

22. Fuentes S, Giralt M. ¿Qué te ocurre..., mama? Barcelona: Ed. Acción Médica, 2006.

23. Epstein N, Baldwin L, Bishop D. The McMaster Family Assessment Device. J Marital Fam Ther 1983;9;171-80. doi: 10.1111/j.1752-0606.1983.tb01497.x

24. Fuentes, S. Comunicació en cáncer: Anàlisi de les preocupacions i dels recursos dels pares recen diagnosticats al comunicar la malaltia als seus fills. (Tesi Doctoral). Barcelona: Universitat Autònoma de Barcelona. 2015.

25. Gessler S, Low J, Daniells E, Williams R, Brough V, Tookman A, et al. Screening for distress in cancer patients: is the distress thermometer a valid measure in the UK and does it measure change over time?. A prospective validation study. Psychooncology 2008; 17:538-47. doi: 10.1002/pon.1273

26. Priede A, Torres M, Hoyuela F, González-Blanch C. El termómetro del distress como prueba de cribado de ansiedad y depresión en pacientes oncológicos recién diagnosticados. Psicooncología 2014;11:31-43. doi: 10.5209/rev_PSIC.2014.v11.n1.44915

27. Zwahlen D, Hagenbuck N, Carley M, Recklitis C, Buchi S. Screening cancer patients' families with the distress thermometer (DT): a validation study. Psychooncology 2008;17:959-66. doi: 10.1002/pon.1320.

28. Kim S, Ko Y, Jun E. The impact of breast cancer on mother-child relationship in Korea. Psychooncology 2012;21 640-6. doi: 10.1002/pon.1941

29. Bugge K, Helseth S, Darbyshire P. Parent's experiences of a Family Support Program when a parent has incurable cancer. J Clin Nurs 2009; 8:3480-8. doi: 10.1111/j.13652702.2009.02871.x.

30. Romer G, Saha R, Haagen M, Pott M, Baldus C, Bergelt C. Lessons learned in the implementation of an innovative consultation and liaison service for children of cancer patients in various hospital settings. Psychooncology 2007;16:138-48. doi: 10.1002/ pon. 1105

31. Fasciano K, Berman H, Moore C, DeFrino B, Jameson R, Kennedy S, et al. When a parent has a cancer: a community based program for school personnel. Psychooncology 2007;16:158-67. doi:10.1002/pon.1148

32. Huang X, O’Connor M, Lee S. School-aged and adolescent children's experience when a parent has non-terminal cancer: a systematic review and meta-synthesis of qualitative studies. Psychooncology 2014;23:493-506. doi: 10.1002/pon.3457

33. Grenklo T, Kreicbergs U, Valdimarsdóttir U, Nyberg T, Steineck G, Fürst C. Self-injury in youths who lost a parent to cancer: nationwide study of the impact of family-related and health-care-related factors. Psychooncology 2014;23:989-97. doi: 10.1002/pon.3515

34. Kopchak D, Burke C. Interaction patterns berween parents with advanced cancer and their adolescent children. Psychooncology 2011; 20:1108-15. doi.10.1002/pon.1831

35. Muñoz MJ, Bernal P. Abordaje de la ansiedad en pacientes diagnosticados de càncer. Psicooncologia 2016;13:227-48. doi:10.5209/PSIC.54434 
36. Rodríguez AM. ¿Cómo explicar a los niños y adolescentes el cáncer avanzado o terminal de sus padres? Psicooncologia 2015; 12:171-82. doi: 10.5209/rev_PSIC.2015.v12. n1.48911

37. Vila L, Font A, Caba L. Estado emocional en mujeres con cáncer de mama: variación experimentada después de una sesión psico-oncológica basada en el counselling y la psicología positiva. Psicooncologia. 2016; 13: 205-25. doi:10.5209/PSIC.54433 